the descriptions of the value of such training, which have appeared in the medical press, have all erred on the side of optimism.

I tender my grateful thanks to Miss Margaret Weight who was in charge of the Department at Paddington Green Children's Hospital in its first years, and kindly prepared the data for this article.

\title{
THE USE OF SODIUM AND MERCURY VAPOUR LAMPS IN OPHTHALMOSCOPY
}

\section{BY}

\section{A. J. Ballantyne \\ GLASGOW}

THE writer has been employing sodium and mercury vapour lamps in clinical work during the past two years, and the advantages of these have been referred to in two communications (Ballantyne, 1937).

The inducement to experiment with these selected lights was provided by the demonstration of various forms of discharge lamps during the visit of Members of the Ophthalmological Society to the Research Department of the G.E.C. at Wembley in the Spring of 1936, and the first lamps used were the laboratory pattern sodium and mercury lamps. These were somewhat expensive, and, in the case of the mercury lamp at least, the luminosity was somewhat low for purposes of ophthalmoscopy.

Subsequently, the "Osira" mercury vapour lamp and the "Philco" sodium vapour lamp, as used for street lighting, were obtained, and these were found to give more than ample illumination.

For convenience in consulting room use, Messrs. Curry and Paxton have, at the writer's suggestion, fitted these two tubes on a single standard, but it would be equally easy to fit either or both of them to an adjustable wall bracket.

The electrical current is taken from the mains supply through a choke coil, and in the standard pattern referred to, the two coils are enclosed in a cabinet on the base. A small shelf or table-top is provided at a convenient height and the switches are attached below this on the upright standard. The lamps are attached to the ends of two jointed arms, adjustable for height, and as the luminous area of the discharge tubes is unnecessarily large for ophthalmoscopy, each is enclosed in a light asbestos-lined metal chimney with a suitable aperture, provided with a gallery in which 
filters may be placed if desired. The reflecting ophthalmoscope is used, either by the direct or by the indirect method.

The lamps are of robust make and have a long life. Replacements cost, for the sodium lamp $32 / 6$, and for the mercury lamp $35 \mathrm{~s}$.

For the information of those who are not familiar with the mercury vapour and sodium vapour lamps, I may state briefly their principal features and indicate some of their uses.

The Mercury Vapour Lamp is, of course, no novelty, and the appearance of the fundus oculi under its light has been described and illustrated by Mayou and, more recently, by Duke-Elder; but it has not come into general use. This, I fancy, is partly

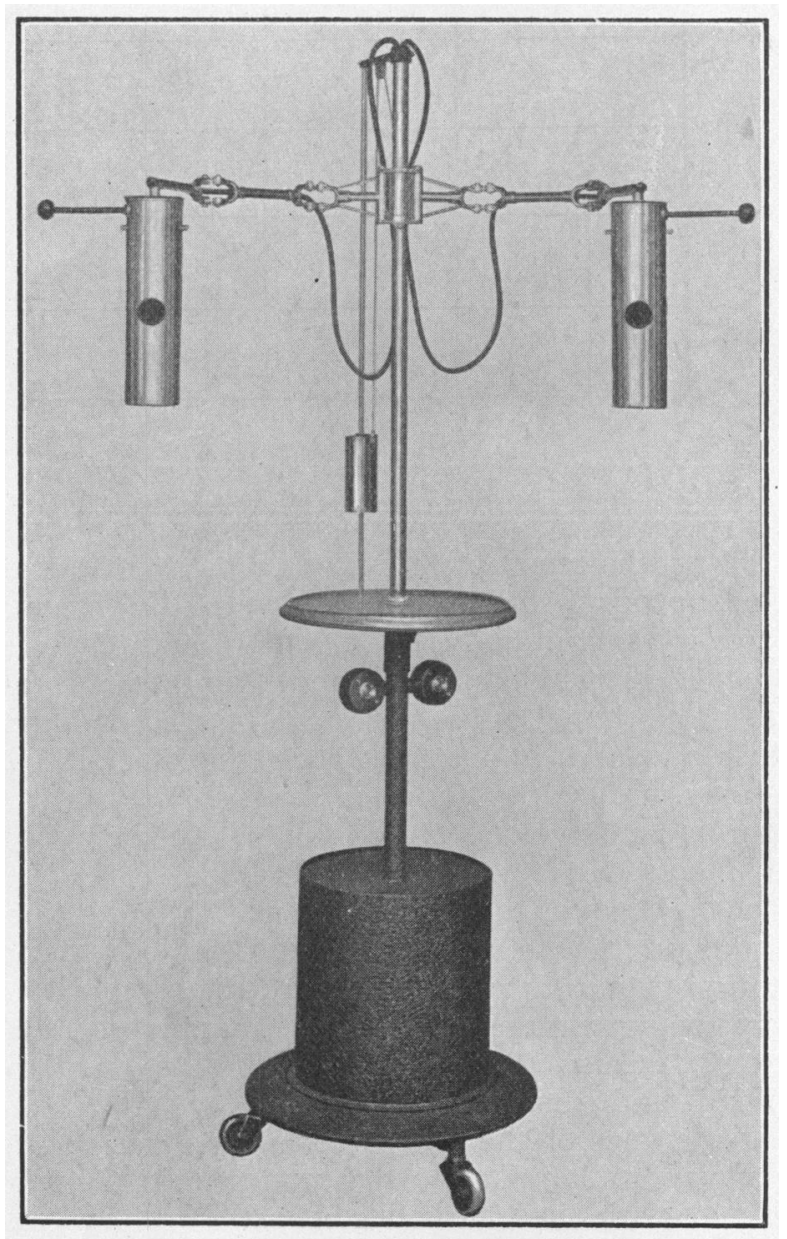


due to the lack of a convenient form of lamp, of durable construction, and not too expensive. These obstacles have now been overcome by the introduction of the discharge lamps used in street and factory lighting.

As will be seen from Fig. 1, the radiation from this lamp includes a good deal of the spectrum, from the violet to the yellow-green; but the greater part of its light is from the blueviolet at 436 millimicrons, and the green at 546 millimicrons.

Since the predominant blue-green radiations are absorbed by the haemoglobin, while the yellow-greens are reflected by the sclera and to some extent by the retinal pigment, the most striking

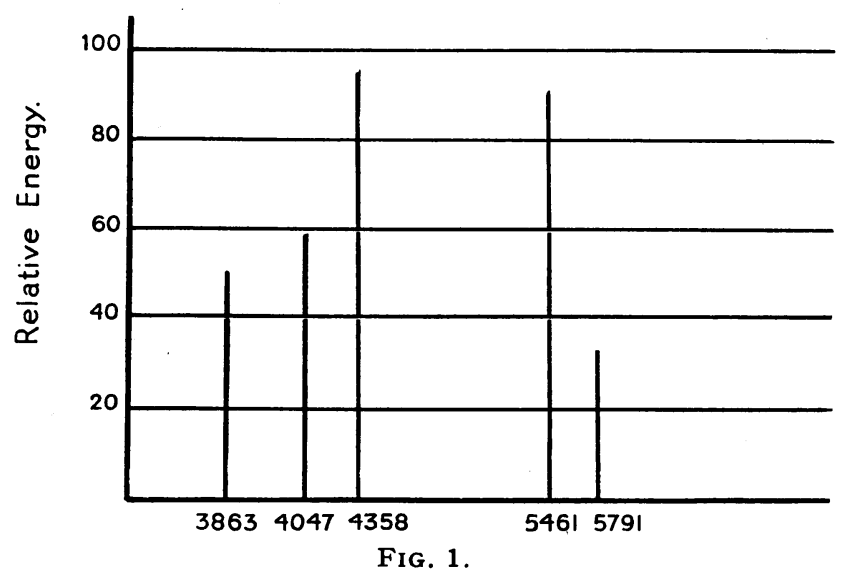

Emission spectrum of mercury vapour lamp.

feature of the ophthalmoscopic picture is the contrast of the chocolate coloured or purplish-black vessels with the greenish-yellow disc and grey-green fundus. The normal yellow spot shows up as an ill-defined brownish patch, about one-third of the area of the macula. The retinal nerve fibres can be traced as they radiate from the disc and arch above and below the macula. When, as in the blonde fundus, the choroidal vessels are seen against a light background, the vessels are darkened and their contrast with the ground increased.

The surface reflexes of the fundus, seen with the ordinary mixed light, are due to the reflection of the short wave components from the anterior limiting membrane, and the light of the mercury vapour lamp, being composed mainly of short waves, exaggerates these reflexes. It aiso gives to the axial reflexes of the vessels a more brilliant and glistening appearance.

The preponderance of the blue-green rays gives to the light of the mercury vapour lamp almost a monochromatic quality, and this, combined with the marked contrast of blood vessels and 
background, renders visible the finest details of the retinal vessel system. Changes in the circumfoveal twigs, or the presence of fine pathological vessels on the disc and elsewhere, are easily discerned. For the same reason, haemorrhages stand out clearly against the fundus, and minute haemorrhages, which escape notice with the ordinary mixed light, are more readily discovered.

Superficial exudates, and retinal exudates in general, are clearly defined and take a greenish colour like that of the optic disc, but changes deep to the retina are not so clearly seen, since the retina is not so transparent to the short waves.

The exaggeration of the retinal surface reflexes may be useful in cases where they give an indication of changes in contour of the retinal surface, as in papilloedema, or oedema in the region of the macula, or where there are traction or pressure folds from various causes. On the other hand, these reflexes are sometimes disadvantageous, as they may conceal or confuse structural changes in a deeper plane.

The unfiltered light of the mercury vapour lamp is not equal to the Vogt red-free lamp for examination of the normal or pathological macula, but for most practical purposes it is a satisfactory substitute for the Vogt lamp, and it has the advantage that it is less disagreeable for the patient, and creates less dazzle for the examiner.

The Sodium Vapour Lamp provides us with a monochromatic light of pure yellow colour, and of high luminosity, well adapted for both direct and indirect ophthalmoscopic examination and for retinoscopy. Its value in the examination and photography of the external aspects of the eye has been emphasised by Kleefeld (1935-1936).

The two most important qualities of this light are, firstly, that it is almost purely monochromatic (Fig. 2) and, secondly; that it

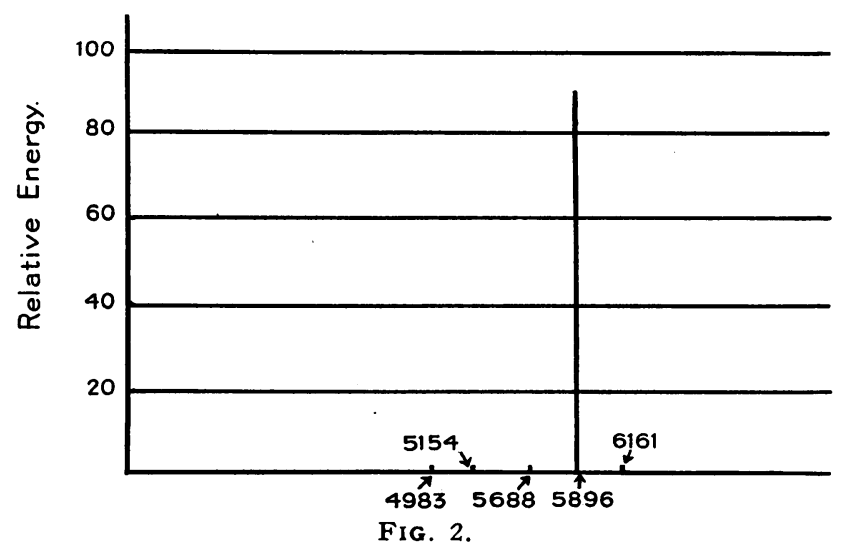

Emission spectrum of sodium vapour lamp. 
is largely absorbed by the blood in the retinal vessels, so that there is a marked contrast between the dark brown colour of these, and the pale greyish-yellow of the fundus. These two properties give the light a notable resolving power, enabling us to follow the retinal vessels to their finest ramifications, and revealing changes in the vessels which might escape notice under the mixed light of the ordinary electric lamp.

In this respect, the light of the sodium vapour lamp resembles that of the mercury vapour lamp; in some cases indeed the latter is preferable, in that the darker tone of the vessels makes recognition of the finest twigs more easy. But whereas a considerable portion of the short wave mercury vapour light is reflected by the anterior limiting membrane and the pigment layer of the retina, the long wave light of the sodium vapour lamp suffers little if any reflection in this way. It is to some extent absorbed by the retinal and uveal pigment, but is largely reflected by the sclera.

Thus, not only does the sodium light not emphasise the surface reflexes of the retina, as does the mercury light; but, where such reflexes are produced by the mixed light of the electric lamp, the sodium light cancels them. The retina, and to a less extent, the choroid, is transparent to the rays, giving us a clearer view of changes in the choroid and the deeper layers of the retina. This can be demonstrated in the case of changes in the walls of the choroidal vessels, in the most minute form of colloid bodies, or other punctate lesions in the lamina vitrea or in the retina, and in sclerotic changes in the finest retinal vessels. The delicate white meshwork, probably gliosis, in the retina, in cases of branch thrombosis of the retinal veins, is more easily detected in the sodium light. It is probably the monochromatic quality of this light which enables us to trace the retinal nerve fibres almost as easily with its help as with the mercury vapour lamp. The fine resolving power of the sodium light was illustrated by the observation that a delicate cotton wool patch in the retina could still be traced by means of this light, after it had disappeared in the ordinary ophthalmoscopic light.

Where appreciation of colour values is essential to the ophthalmoscopic picture, as in the macular changes of myopia, including choroidal atrophy, pigmentation and haemorrhage, neither the sodium nor the mercury light is so useful as the mixed light. Pigment is more transparent to the sodium light than to mixed light, hence, retinal pigment patches or the pigment ring surrounding the disc assume a greyish colour. A melanoma of the choroid disappears under the sodium light.

When the choroidal vessel pattern is seen against a light background, as in the blonde or albinotic fundus, the sodium light enhances the visibility of the choroidal vessels by contrast with 
their background, but in the tessellated brunette fundus, the vessel pattern disappears, owing to the approximately equal absorption of the light by the choroidal vessels and the intervening pigment.

The elimination of surface reflexes from the retina is one of the most interesting and valuable properties of the sodium vapour light. These reflexes may in any case conceal delicate changes in the retina, but this has been most frequently observed in cases presenting oedema of the disc, the surrounding retina or the macular area. In these circumstances, one often sees reflexes which are pathological in their number, extent or formation. When the sodium light is used, and the surface reflexes are eliminated, one is often able to see fine retinal exudates, such, for example, as minute specks arranged in clusters or radiating from the macula. In other cases it reveals retinal lines such as the double-contour lines of Vogt, which are obviously on a deeper plane than the surface reflexes, though the latter may have had the same linear arrangement.

Gunn's dots, which are easily seen in the ordinary ophthalmoscopic light, and are rendered still more striking by the mercury vapour light, disappear under the sodium vapour light. This seems to be clear evidence that, whatever may be the histological basis of the dots, they are very superficial and cause elevation of the anterior limiting membrane of the retina ; but are as colourless and transparent as the tissues of the retina.

Each of these lights has a limited sphere of usefulness, but in the absence of differential staining of the living retina, they have an obvious value. Used in conjunction with, and as a supplement to the ordinary form of illumination, they are a very welcome addition to our resources in ophthalmoscopy, and it is a source of satisfaction that they are now available in a handy form for clinical work.

\section{REFERENCES}

Ballantyne, A. J.-(a) Bimicroscopy of the fundus oculi. Trans. Royal MeaicoChir. Soc. Glasgow, Vol. XXXI, p. 74, 1937

(b) Modern Methods in Ophthalmoscopy. Trans. Ophthal. Soc. U.K., Vol. LVII, p. 2731937

KLEefeld, G. - Exploration de l'oeil au moyen de la lampe au Sodium. Bull. de la Soc. d'Ophtal. de Paris, December, 1935.

Examen objectif de l'oeil au msyen de la lumière monochromatique jaune (lampes au Sodium). Bull. de la Soc. Belge d'Ophtal., No. 72, p. 24, $193 E$. 